\title{
Riboflavin Transporter 1 Deficiency Caused by a Homozygous Single Exonal Deletion of SLC52A1
}

\author{
Urim Kang, MD1, Da Hae Yang, MD², Sang Ook Nam, MD³,4 Yun-Jin Lee, MD,4, Gyu Min Yeon, MD \\ ${ }^{1}$ Department of Pediatrics, Kosin University Gospel Hospital, Kosin University College of Medicine, Busan, Korea \\ ${ }^{2}$ Department of Laboratory Medicine, Kosin University Gospel Hospital, Kosin University College of Medicine, Busan, Korea \\ ${ }^{3}$ Department of Pediatrics, Pusan National University Children's Hospital, Pusan National University School of Medicine, Yangsan, Korea \\ ${ }^{4}$ Research Institute for Convergence of Biomedical Science and Technology, Pusan National University Yangsan Hospital, Yangsan, Korea
}

Received: July 9, 2020

Revised: August 6, 2020

Accepted: August 12, 2020

Corresponding author:

Gyu Min Yeon, MD

Department of Pediatrics, Kosin

University Gospel Hospital, Kosin

University College of Medicine, 262

Gamcheon-ro, Seo-gu, Busan

49267, Korea

Tel: +82-51-990-5212

Fax: +82-51-990-3005

E-mail:ygmcu@hanmail.net
Riboflavin (vitamin B2) is the solitary precursor for the biologically active cofactors known as the flavin mononucleotide (FMN) and flavin adenine dinucleotide (FAD) molecules [1]. These cofactors are required in oxidation-reduction (redox) reactions and act as cofactors for the electron transfer flavoprotein (ETF) and its dehydrogenase (ETFDH) [2]. The ETF and ETFDH form electron transport pathways for at least 12 mitochondrial flavoprotein dehydrogenases involved in amino acid, fatty acid, and choline metabolism [3]. Variations of the ETF or ETFDH cause multiple acyl-CoA dehydrogenation deficiencies (MADDs), and riboflavin metabolism or transport genetic defects can also cause MADD or varying degrees of progressive neurodegenerative diseases such as riboflavin transporter deficiency (RTD) [3].

There are three human riboflavin transporter (RFVT) homologs: RFVT 1 to 3, encoded by genes SLC52A1 to SLC52A3, respectively [4]. RFVTs are widely distributed in the body and SLC52A1 is highly expressed in the placenta and intestine [3]. SLC52A2 is rather ubiquitously expressed, mainly in the brain, and, although SLC52A3 is most highly expressed in the testes, which also expressed in the intestine and prostate [3]. There are three types of RTDs and these are caused by autosomal dominant, heterozygous (SLC52A1 [OMIM: 615026]), and autosomal recessive (AR), biallelic (SLC52A2 [OMIM: 614707] and SLC52A3 [OMIM: 211500 and 211530]) mutations corresponding to RTD1, RTD2, and RTD3, respectively [4]. Although 24 cases of RTD2 and 49 cases of RTD3 have been reported, only two cases of RTD1 have been reported so far [3-5]. The first case was published in 2007, and in 2011 a maternal RTD was identified, caused by a heterozygous microdeletion of the maternal SLC52A1, with transient neonatal-onset MADD [5]. The second case reported a transient neonatal-onset riboflavin-responsive MADD caused by maternal riboflavin deficiency, with detection of heterozygous intronic variations of both the maternal and fetal SLC52A1 [3]. To the best of our knowledge, this is the first case report of a homozygous exon 3 deletion in SLC52A1 in an infant in the absence of maternal RTD. It may have occurred due to AR inheritance via paternal heterozygous exonal deletions and a $25 \%$ decrease in the concentration of DNA in exon 3 in the mother, a mosaic heterozygous exon 3 deletion that could affect the homozygous deletion of exon 3 in the patient was considered.

A previously healthy 4-month-old girl present-

Copyright (C) 2020 Korean Child Neurology Society

This is an Open Access article distributed under the terms of the Creative Commons Attribution Non-Commercial License (http://creativecommons.org/licenses/by-nc/4.0/) which permits unrestricted non-commercial use, distribution, and reproduction in any medium, provided the original work is properly cited. 
ed to our hospital with repeated seizures. Two days prior to presenting to the hospital, while she was asleep in the car on a family trip, she experienced left eyeball deviation accompanied by a generalized tonic-clonic seizure lasting for 2 minutes. On the day of her visit, when she woke up from a nap, she experienced, for 1 minute, a focal clonic seizure of the right extremities. Her consciousness recovered well and no febrile illness was observed before and after these two episodes. At the time of the hospital visit, her blood pressure, pulse rate, respiratory rate, body temperature, and oxygen saturation were $80 / 50 \mathrm{~mm} \mathrm{Hg}, 124$ beats/minute, 32 breaths $/ \mathrm{min}, 36.9^{\circ} \mathrm{C}$, and $97 \%$, respectively.

The infant was born, by cesarean section, at the gestational age of $38^{+1}$ weeks, weighing $3,300 \mathrm{~g}$. There were no prenatal or perinatal problems. Tandem mass spectroscopy was normal and she showed normal growth and development. There was no family history of seizures or other neurologic diseases. Her body weight, height, head circumference, and neurologic examination were within the normal range.

Blood biochemical analysis revealed white blood cell 10,840/ $\mathrm{mm}^{3}$, hemoglobin $11.5 \mathrm{~g} / \mathrm{dL}$, platelet $467,000 / \mathrm{mm}^{3}$, C-reactive protein $0.063 \mathrm{mg} / \mathrm{dL}$, aspartate aminotransferase $59 \mathrm{IU} / \mathrm{L}$, alanine aminotransferase $61 \mathrm{IU} / \mathrm{L}$, and glucose $121 \mathrm{mg} / \mathrm{dL}$. Electrolytes levels were normal. Venous blood gas analysis showed compensated metabolic acidosis with pH 7.275, PCO2 $36.3 \mathrm{~mm} \mathrm{Hg}$, bicarbonate $17.0 \mathrm{mmol} / \mathrm{L}$, base excess -8.4 , and elevated ammonia $170 \mu \mathrm{mol} / \mathrm{L}$. No specific lesion was observed in the brain magnetic resonance imaging, and electroencephalography (EEG) showed interictal epileptiform discharges (IEDs) over the midline central region.

Hyperammonemia was considered due to the transient elevation after the seizure. Epilepsy was the suspected diagnosis and we started administering oxcarbazepine. After an intravenous infusion of $5 \%$ dextrose water with electrolytes throughout the day, serum ammonia decreased to $94 \mu \mathrm{mol} / \mathrm{L}$. However, after the discontinuation of the dextrose fluid with full enteral feeding, ammonia level became elevated, at $208 \mu \mathrm{mol} / \mathrm{L}$ on day 2 . Treatment of hyperammonemia was initiated, with fasting for 24 hours and sufficient administration of glucose to prevent additional hyperammonemia via protein catabolism. In addition, nitrogen scavenger arginine was administered intravenously and carnitine was administered to buffer toxic acyl-CoA intermediates. On day 3, serum ammonia decreased to $58 \mu \mathrm{mol} / \mathrm{L}$ and feeding resumed. Serum ammonia was maintained as 52 to $74 \mu \mathrm{mol} / \mathrm{L}$ for a month thereafter. A metabolic workup for serum and urine taken to find the cause of hyperammonemia showed normal results. Gene panel tests for inborn errors of metabolism and epilepsy did not detect any gene mutations. A variation was observed on partial exome sequencing for 5,447 genes using copy number variation $(\mathrm{CNV})$ analysis based on the human GRCh37: 17p13.2(4936243-4937911) × 1, 1.7kb, containing SLC52A1 exons 2, 3, 4, 5. During the CNV analysis, statistically meaningful values were derived by comparing the sequencing depths with other sample data; the eXome-Hidden Markov Model (XHMM) algorithm was used for the analysis (PMID: 23040492). Subsequent droplet digital polymerase chain reaction (ddPCR) revealed a homozygous exon 3 deletion in SLC52A1 and heterozygous deletions in exons 1, 2, 4, and 5 (Fig. 1). Finally, the patient was diagnosed with RTD1 and started taking additional riboflavin. Targeted CNV analysis and ddPCR of her parents revealed heterozygous deletions in exons $1-5$ of SLC52A1 in her father and a $25 \%$ decrease in the concentration of DNA in exon 3, which was suggestive of a mosaic heterozygous exon 3 deletion that could affect the homozygous deletion of exon 3 (Fig. 1). Her brother had no mutation. Thus, the proband's phenotypic characteristics may be attributed to the homozygous single exonal deletion of SLC52A1 with a probable underlying AR inheritance. This study was approved by the Institutional Review Board (IRB) of Kosin University Gospel Hospital (IRB no., 2020-06-014). Informed consent was waived due to the retrospective nature of the study.

Since then, she has been seizure-free and her development has been normal. We continued oxcarbazepine therapy because of occasional IEDs identified in the EEG, which may be caused by mildly sustained hyperammonemia, and might lead to seizure recurrence. Serum ammonia remained between 57 and $61 \mu \mathrm{mol} / \mathrm{L}$ for about 18 months. She is currently 22 months old, with a head circumference in the 5 th percentile, and takes $100 \mathrm{mg}$ of riboflavin, $900 \mathrm{mg}$ of carnitine, and $96 \mathrm{mg}$ of oxcarbazepine per day. After increasing riboflavin dosing to $100 \mathrm{mg}$ per day, her serum ammonia dropped to $44 \mu \mathrm{mol} / \mathrm{L}$; therefore, we planned to discontinue oxcarbazepine treatment if her serum ammonia and EEG findings were maintained within the normal limits. RFVT3 plays a major role when riboflavin is absorbed into the epithelial cells of the small intestine. Afterward, riboflavin migrates to the bloodstream, either assisted by RFVT1 or RFVT2, or directly through conversion to the FAD coenzyme via FMN [3]. RFVT2 plays a major role in blood-brain barrier passage, for reaching the brain tissue [3]. When the maternal riboflavin passes through the placenta, RFVT1 becomes the only way to the fetus [3]. If a maternal RTD1 exists, fetal MADD may occur, sometimes causing fetal death in fatal cases [3]. When RTD1 presents only in a child, transport of riboflavin from small intestinal epithelial cells into the blood would be partially impaired, because of the transportation by RFVT2 or direct passing through conversion to FAD [1]. This is why an RTD1 without maternal RTD1 displays relatively mild symptoms and an infant with RTD1 can be treated effectively. In this case, RTD1 was diag- 
A

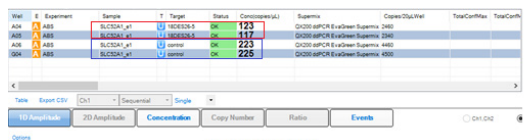

3

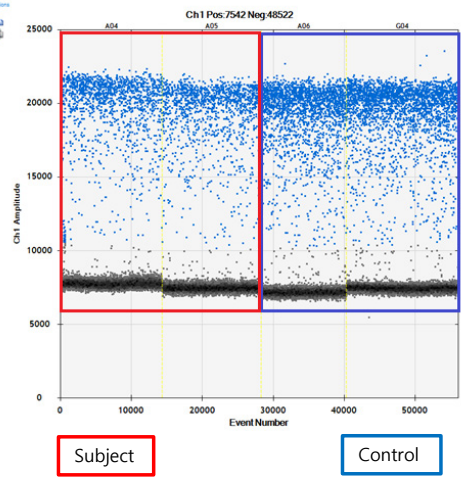

(D)
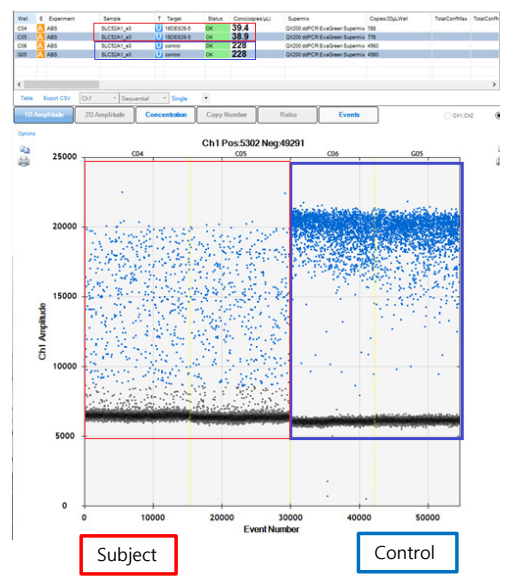

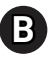

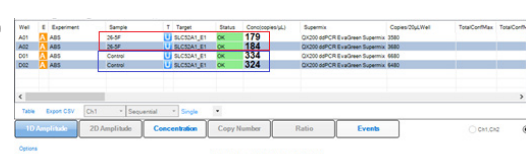

$\stackrel{3}{3}$

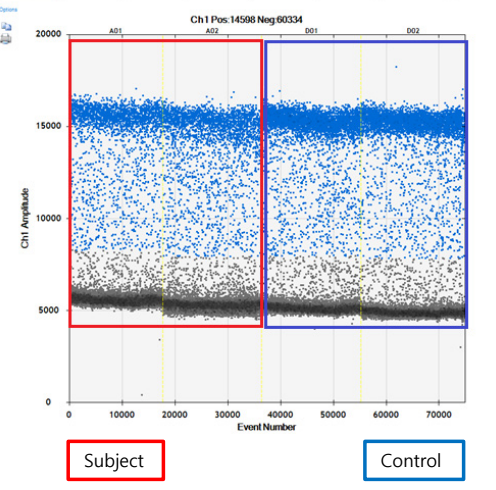

E

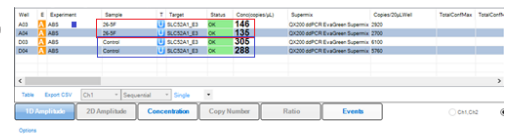

2000

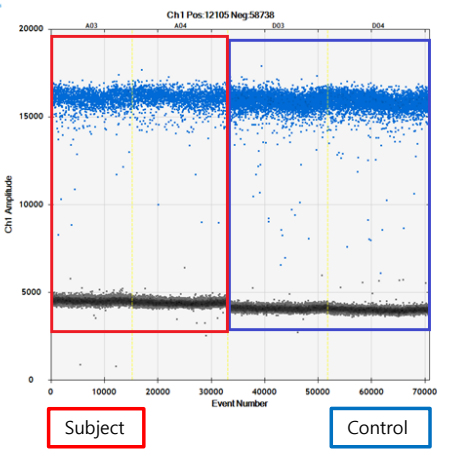

C

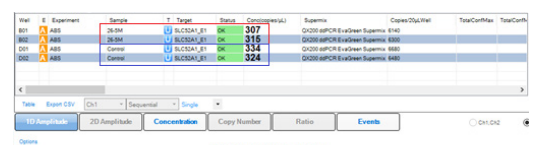

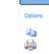

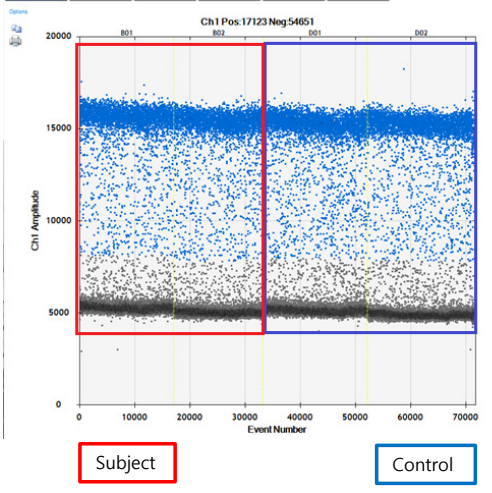

F
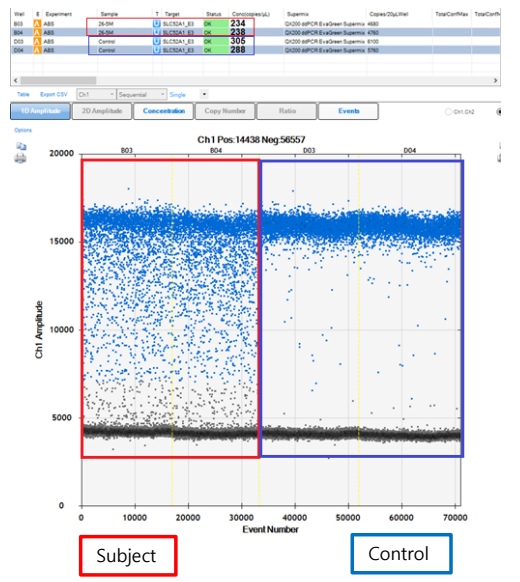

Fig. 1. The droplet digital polymerase chain reaction results of exons 1 and 3 of SLC52A1. DNA concentrations of exon 1 in the (A) patient and (B) her father were approximately half of that of the control, and this is consistent with a heterozygous deletion. (C) The DNA concentration of exon 1 in her mother was equal to that of the control, which means that there was no deletion. (D) The DNA concentrations of exon 3 were 39.4 and 38.9 copies $/ \mu \mathrm{L}$ in the patient and 228 and 228 copies $/ \mu \mathrm{L}$ in the control, which is consistent with a homozygous deletion. (E) Her father's DNA concentration of exon 3 was approximately half of that of the control, which is consistent with a heterozygous deletion. (F) The DNA concentrations of exon 3 were 234 and 238 copies/ $\mu \mathrm{L}$ for her mother and 305 and 288 copies/ $\mu \mathrm{L}$ for the control. These results may be due to the mosaic heterozygous deletion of exon 3 , which may have affected the patient's homozygous deletion.

nosed in an infant, without maternal riboflavin deficiency, and gene testing suggested paternal inheritance in the initial diagnosis. Her father was healthy and had no symptoms related to this mutation. The homozygous deletion in exon 3 of SLC52A1, which was only found in the proband, may have accounted for her symptoms, especially when her parents were asymptomatic. In two previously reported cases of RTD1, both mothers were also symptom-free and the infant with RTD1 were treated until 3 years of age. However, in our case, the mutation was a homozygous deletion, which may have influenced a different clinical course of the disease. While RTD1 may be a transient disorder with a heterozygous mutation, further studies pertaining to the clinical courses are needed.

Children with RFVT1 deficiency due to the heterozygous mutation of SLC52A1 may be asymptomatic, but maternal RFVT1 deficiency should be considered when a neonate has hyperammonemia, metabolic acidosis, or a convulsive history of unknown etiology. The neonate and the mother can be healthy if treated with proper supplementation of riboflavin. Children with RFVT1 deficiency due to the homozygous variation of SLC52A1 can present with hyperammonemic seizures and it is important for them to take sufficient riboflavin to control hyperammonemia, and in turn to be seizure-free with normal development.

\section{Conflicts of interest}

No potential conflict of interest relevant to this article was reported. 


\section{ORCID}

Urim Kang, https://orcid.org/0000-0002-6411-8484

Gyu min Yeon, https://orcid.org/0000-0002-1537-8554

\section{Author contribution}

Conceptualization: UK, DHY, and GMY. Data curation: UK, DHY, and YJL. Formal analysis: SON and GMY. Methodology: UK, DHY, SON, and GMY. Project administration:DHY, SON, YJL, and GMY. Visualization: DHY and YJL. Writing-original draft: UK and GMY.

\section{Acknowledgements}

We would like to thank Dr. Chang Ahn Seol (Genome Research Center, GC Genome) for analysing gene mutation of the patient.

\section{References}

1. Powers HJ. Riboflavin (vitamin B-2) and health. Am J Clin Nutr 2003;77:1352-60.

2. Houten SM, Wanders RJ. A general introduction to the biochemistry of mitochondrial fatty acid $\beta$-oxidation.J Inherit Metab Dis 2010;33:469-77.

3. Mosegaard S, Bruun GH, Flyvbjerg KF, Bliksrud YT, Gregersen $\mathrm{N}$, Dembic M, et al. An intronic variation in SLC52A1 causes exon skipping and transient riboflavin-responsive multiple acylCoA dehydrogenation deficiency. Mol Genet Metab 2017; 122:182-8.

4. O'Callaghan B, Bosch AM, Houlden H. An update on the genetics, clinical presentation, and pathomechanisms of human riboflavin transporter deficiency. J Inherit Metab Dis 2019; 42:598-607.

5. Ho G, Yonezawa A, Masuda S, Inui K, Sim KG, Carpenter K, et al. Maternal riboflavin deficiency, resulting in transient neonatal-onset glutaric aciduria type 2 , is caused by a microdeletion in the riboflavin transporter gene GPR172B. Hum Mutat 2011;32:E1976-84. 